\title{
Antibiotics for coughing in general practice: a questionnaire study to quantify and condense the reasons for prescribing Samuel Coenen*1, Barbara Michiels ${ }^{1}$, Paul Van Royen ${ }^{1}$, Jean-Claude Van der Auwera ${ }^{1}$ and Joke Denekens ${ }^{1}$
}

Address: ${ }^{1}$ Centre for General Practice, University of Antwerp-UIA, Antwerp, Belgium and 2Epidemiology and Social Medicine, University of Antwerp-UIA, Antwerp, Belgium

E-mail: Samuel Coenen* - samuel.coenen@ua.ac.be; Barbara Michiels - barbara.michiels@ua.ac.be; Paul Van Royen - paul.vanroyen@ua.ac.be; Jean-Claude Van der Auwera - jean-claude.vanderauwera@ua.ac.be; Joke Denekens - joke.denekens@ua.ac.be

${ }^{*}$ Corresponding author

Received: I 5 July 2002

BMC Family Practice 2002, 3:16

Accepted: 9 September 2002

This article is available from: http://www.biomedcentral.com/I47I-2296/3/16

(c) 2002 Coenen et al; licensee BioMed Central Ltd. This article is published in Open Access: verbatim copying and redistribution of this article are permitted in all media for any non-commercial purpose, provided this notice is preserved along with the article's original URL.

\begin{abstract}
Background: Antibiotics are being overprescribed in ambulant care, especially for respiratory tract infections (RTIs). Gaining insight into the actual reasons for prescribing remains important for the design of effective strategies to optimise antibiotic prescribing. We aimed to determine items of importance for the antibiotic prescribing decision and to make them operational for an intervention trial.
\end{abstract}

Methods: A postal questionnaire based upon focus group findings was sent to 316 Flemish general practitioners (GPs). On a verbal rating scale the GPs scored to what extent they consider the questionnaire items in decision making in case of suspected RTI in a coughing patient and how strongly the items support or counter antibiotic treatment. Factor analysis was used to condense the data. The relative importance of the yielded operational factors was assessed using Wilcoxon Matched Pairs test.

Results: $59.5 \%$ completed the study. Response group characteristics (mean age: 42.8 years; $65.9 \%$ men) approximated that of all Flemish GPs. Participants considered all the items included in the questionnaire: always the operational factor 'lung auscultation', often 'whether or not there is something unusual happening' - both medical reasons - and to a lesser extent 'non-medical reasons' $(\mathrm{P}<0.001)$. Non-medical as well as medical reasons support antibiotic treatment, but non-medical reasons to a lesser extent $(P<0.001)$.

Conclusion: This study quantified, condensed and confirmed the findings of previous focus group research. Practice guidelines and interventions to optimise antibiotic prescribing have to take nonmedical reasons into account.

\section{Background}

Antibiotics are being overprescribed in ambulant care,[1] especially for respiratory tract infections (RTIs).[2] For this prescribing decision different types of determinants are already highlighted. [3-6] However, gaining insight into the actual reasons for context specific prescribing re- 
mains important to design effective strategies to optimise antibiotic prescribing.[7]

In general practice, medical decisions (concerning RTIs) are prompted most often by complaints about coughing: 169 times per 1000 patients per year for a new illness episode. [8] Since there is no evidence base for the prescription of antibiotics for coughing in case of suspected RTI,[9] and since antibiotic prescribing results in financial costs to the patient and society, adverse effects and development of bacterial resistance,[10] we explored the diagnostic and therapeutic decisions of Flemish general practitioners (GPs) regarding adult patients who consult them with complaints about coughing by means of focus groups.[11] We found medical as well as non-medical reasons for antibiotic prescriptions in case of suspected RTI.[12] Our hypotheses on Flemish GPs' decisions were in line with previous research. The differentiation between RTIs, e.g. acute bronchitis and pneumonia, could not be achieved with certainty on the basis of medical history and clinical examination:[13] i.e. medical reasons. Dealing with this diagnostic uncertainty, GPs' decisions were directed at whether or not to prescribe antibiotics.[14] Determinants playing an important role in this decision are physician related, e.g. having missed pneumonia once, or patient related, e.g. patient expectations:[15] i.e. non-medical reasons.

Since it is time for action, [16] besides a better understanding of the actual determinants for context specific prescribing of antibiotics, we also have to make them operational for the design of an intervention. Therefore, we aimed to quantify and to condense the determinants generated in the focus group study. By means of this postal questionnaire study in Flemish general practice, we assessed to what extent Flemish GPs consider those determinants in decision making in case of suspected RTI in a coughing patient and how strongly the determinants support or counter antibiotic treatment.

\section{Methods}

Design

We performed an explanatory study comparing GPs' responses from a self-administered questionnaire based upon focus group findings. ${ }^{12}$

\section{Setting and sample}

We approached Flemish GPs who were willing to participate in previous studies of our research unit $[12,17]$. The questionnaire was sent to this selected group by mail early September 1999. A reminder was sent to all non-responders two weeks later. Responses were accepted until the end of September 1999. The survey was pilot tested.
Of the 316 GPs originally selected to be in the sample, 7 were no longer practising, 5 returned surveys with more than $20 \%$ of items unanswered, 116 failed to respond before the end of September 1999, leaving 188 GPs who completed the survey. The overall response rate was $59.5 \%$.

\section{Instrument}

To assess the importance of determinants for the antibiotic prescribing decision the GPs were sent one questionnaire in two parts, one (Q1) assessing to what extent they consider these determinants in decision making in case of suspected RTI in a coughing patient, and another (Q2) assessing how strongly these determinants support or counter antibiotic treatment. The response set for each item was a 5-point verbal rating scale (VRS). For Q1 the VRS ranged from 1 (never), 2 (seldom), over 3 (sometimes), to 4 (often) and 5 (always), for Q2 from 2 (strongly in favour), 1 (in favour), over 0 (neutral), to - 1 (against) and 2 (strongly against). 'See additional file: Questionnaire.doc for the original questionnaire'

Focus groups, exploring the determinants of GPs' diagnostic and therapeutic decisions in adult patients with complaints about coughing, provided the items proposed in the questionnaire.[12] The main categories at which we ordered the determinants were: epidemiology, e.g. an influenza epidemic, prior knowledge of the patient, e.g. he/she is smoking, symptoms, e.g. a sputum producing cough, clinical signs, e.g. a normal lung auscultation, patient related non-medical determinants, e.g. patient's demand for antibiotics, physician related non-medical determinants, e.g. having missed pneumonia once. There were also: first impression, e.g. patient looks very ill, laboratory results, e.g. normal erythrocyte sedimentation rate, and natural course, e.g. the illness is worsening.

For the questionnaire (Q1 and Q2) items were chosen from all the above categories to include a meaningful selection of all the issues mentioned in the focus group study. Concerning their content the items were to be manageable in daily practice and to determine the decision whether or not to prescribe antibiotics. Therefore no issues about laboratory results were included: blood or sputum analysis, or X-ray examination are seldom performed in - Flemish [Coenen, unpublished] - general practice in case of suspected RTI.[8] Determinants only used to confirm other possible diagnoses with coughing as principal complaint, e.g. risk factors for pulmonary oedema, were also excluded. The items were to allow clear and brief formulation as well. Five physicians staffing our Centre, including two authors of the report (PVR and SC) selected the issues in the decision to prescribe in consensus and rephrased them into questionnaire items faithful to the original formulation. 


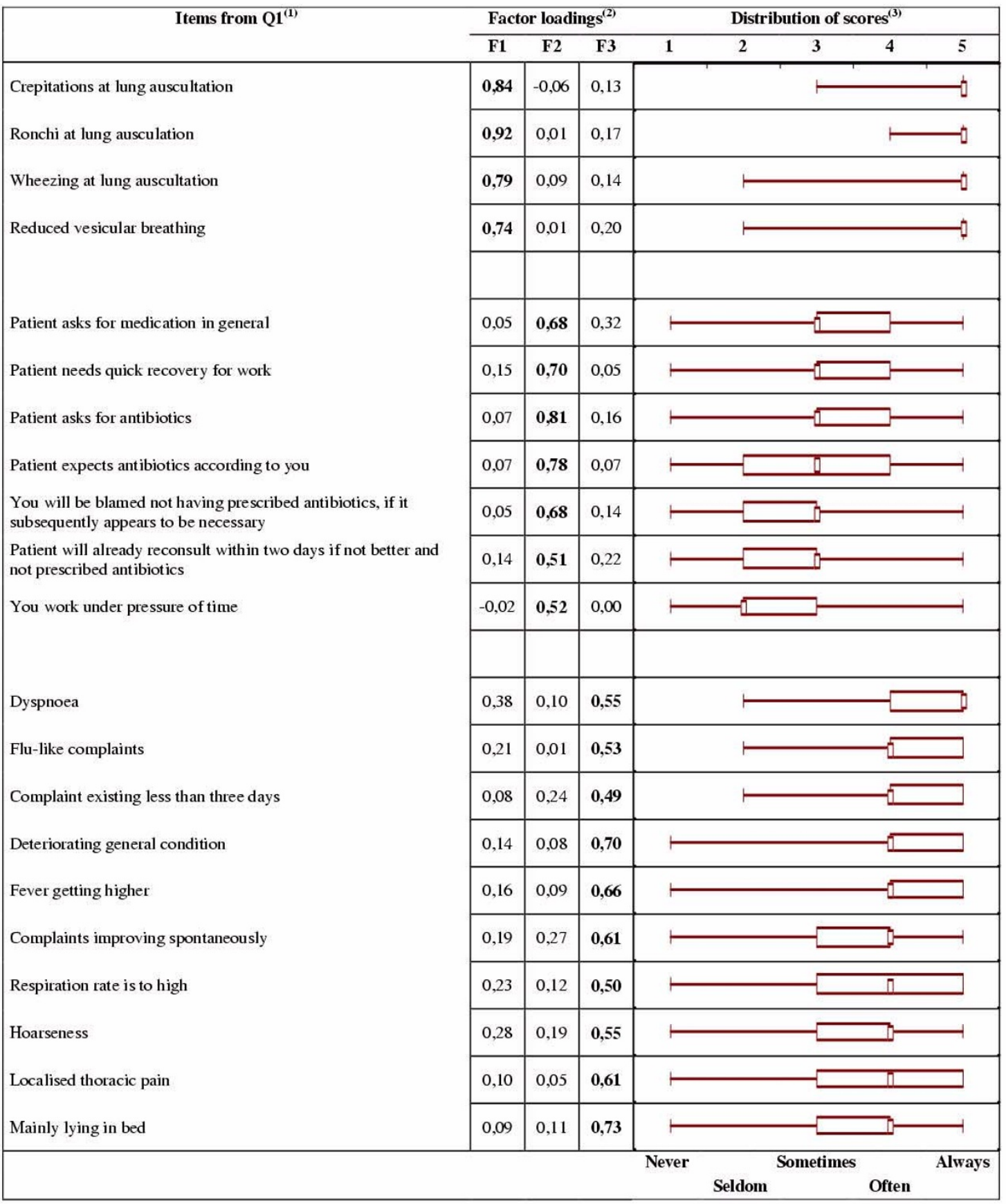

Figure I

Items from part I of the questionnaire (QI)(I): distribution of scores and factor loadings per yielded factor (I) Part I of the questionnaire $(\mathrm{Q} I)$ is assessing how strong the questionnaire items argue in favour or against antibiotic treatment in case of suspected RTI in a coughing patient. Only items with factor loading $>.40$ to the yielded factor are presented. (2) Factor loadings to the yielded factors are presented. Factor I (FI) includes all the items relating to the lung auscultation. Factor 2 (F2) includes only items relating to non-medical reasons, either patient or physician related. Factor 3 (F3) includes items determining whether or not there is something unusual happening. (3) The small box represents the median, the larger box the interquartile range, the wiskers the scoring range. 


\section{Analysis}

All statistical analyses were performed using Statistica 5.1 (StatSoft, Inc., Tulsa, OK, USA). To make the selected issues operational for an intervention trial, exploratory factor analyses on the questionnaire's items in Q1 and Q2 were performed, using the principal axis method and varimax normalised rotation. The relative importance of the operational factors yielded was assessed using Wilcoxon Matched Pairs test. For comparison of ordinal variables between two groups, the Mann-Whitney test was used.

\section{Results}

The mean age (SD) of the GPs was 42.8 years $(7.7), 65.9 \%$ were men. $46.9 \%$ of the GPs worked single-handed. GPs were predominantly rewarded by fee for service; $24.9 \%$ had more than 120 patient encounters per week.

\section{Considering the determinants in decision making}

Assessing to what extent GPs consider the determinants for antibiotic prescribing in decision making (Q1), on average GPs considered all 42 items. Factor analysis suggests groups of variables whose values are similar, in this case GPs' responses to Q1 items. Factor analysis of all items from Q1 yielded three factors, i.e. groups of items which GPs considered similarly, explaining $33 \%$ of the variance (Fig. 1). Factor 1 included all the items relating to the lung auscultation. Factor 2 included only items relating to nonmedical reasons, either patient or physician related. Factor 3 included items determining whether or not there is something unusual happening. Each factor grouping had good internal consistency, with Cronbach $\alpha$ equal to .90 for factor $1, .86$ for factor 2 and .87 for factor 3 .

The median (interquartile range) scores as defined by factor analysis were 5.0 (from 5.0 to 5.0) for factor 1, lung auscultation, 3.0 (form 2.8 to 4.0 ) for factor 2, non-medical reasons, and 4.0 (from 4.0 to 5.0 ) for factor 3, unusual or not.

Using Wilcoxon Matched Pairs test to compare the scores of the factors - scores of factor 1 did not approximate a normal distribution - the differences between all three factors are significant at $P<0.001$ (Fig. 2). Since the differences between the scores approximate a normal distribution, this test is almost as powerful as the $t$-test.

Of course, GPs also considered items from Q1 not presented in figure 1 (factor loading $\leq .40$ to the yielded three factors); always (median = 5) whether the patient has fever, is coughing up sputum and whether the sputum is coloured, whether the patient is looking ill and whether he/she has a medical history of COPD or smoking; often (median $=4$ ) whether the coughing is frequent or started suddenly and whether the patient consults for the first time with this complaint, is saying he/she is feeling ill, is older than 60 years of age, tried self-management first, is known to you or has a red throat, as well as whether there is an RTI epidemic and whether the patient rapidly consults and will reconsult if not better; sometimes (median = 3 ) whether the patient is compliant, or is recovering slowly even under antibiotic treatment; seldom (median = 2) whether the patient is visited at home or that you make the patient reconsult anyway after 3 to 4 days. For most items the interquartile range was 1 .

\section{In favour or against antibiotics}

Assessing how strongly the determinants for antibiotic prescribing support or counter antibiotic treatment (Q2), none of the 63 items is strongly in favour or against antibiotic treatment. Factor analysis of all items from Q2 yielded two factors, i.e. groups of items which according to the GPs support antibiotic treatment similarly. The factors included items expressing a need for antibiotic treatment, and no need for antibiotic treatment respectively. This confirmed our construction of Q2.

\section{In favour}

Factor analysis of all 37 items that support antibiotic treatment according to their mean and sumscore, yielded two factors, i.e. groups of items which according to the GPs are equally in favour of antibiotic treatment, explaining $24 \%$ of the variance (Fig. 3). Factor 1 only included items relating to medical reasons, either from the lung auscultation or determining whether or not there is something unusual happening, factor 2 only included items relating to nonmedical reasons, either patient or physician related. Each factor grouping had good internal consistency, with Cronbach $\alpha$ equal to .82 for factor $1, .83$ for factor 2 .

The median (interquartile range) scores as defined by factor analysis were 1.0 (from 0.5 to 1.0 ) for factor 1 , medical reasons and 0.0 (from 0.0 to 1.0 ) for factor 2, non-medical reasons. Using Wilcoxon Matched Pairs test the scores of the two factors differed significantly at $P<0.001$ (Fig. 4).

\section{Against}

Factor analysis of all 26 items that fail to support antibiotic treatment according to their mean and sumscore, yielded only one factor, i.e. group of items which according to the GPs are equally against antibiotic treatment, explaining $17 \%$ of the variance (Fig. 5). The factor only included items expressing no need for antibiotic treatment, either medical or non-medical. Factor grouping had good internal consistency, with Cronbach $\alpha$ equal to .80 .

The median (interquartile range) score as defined by factor analysis was -1.0 (from -1.0 to -0.5 ). Using Wilcoxon Matched Pairs test the score of this factors differed significantly at $P<0.001$ form the scores of the two factors in favour of antibiotics (Fig. 4). 


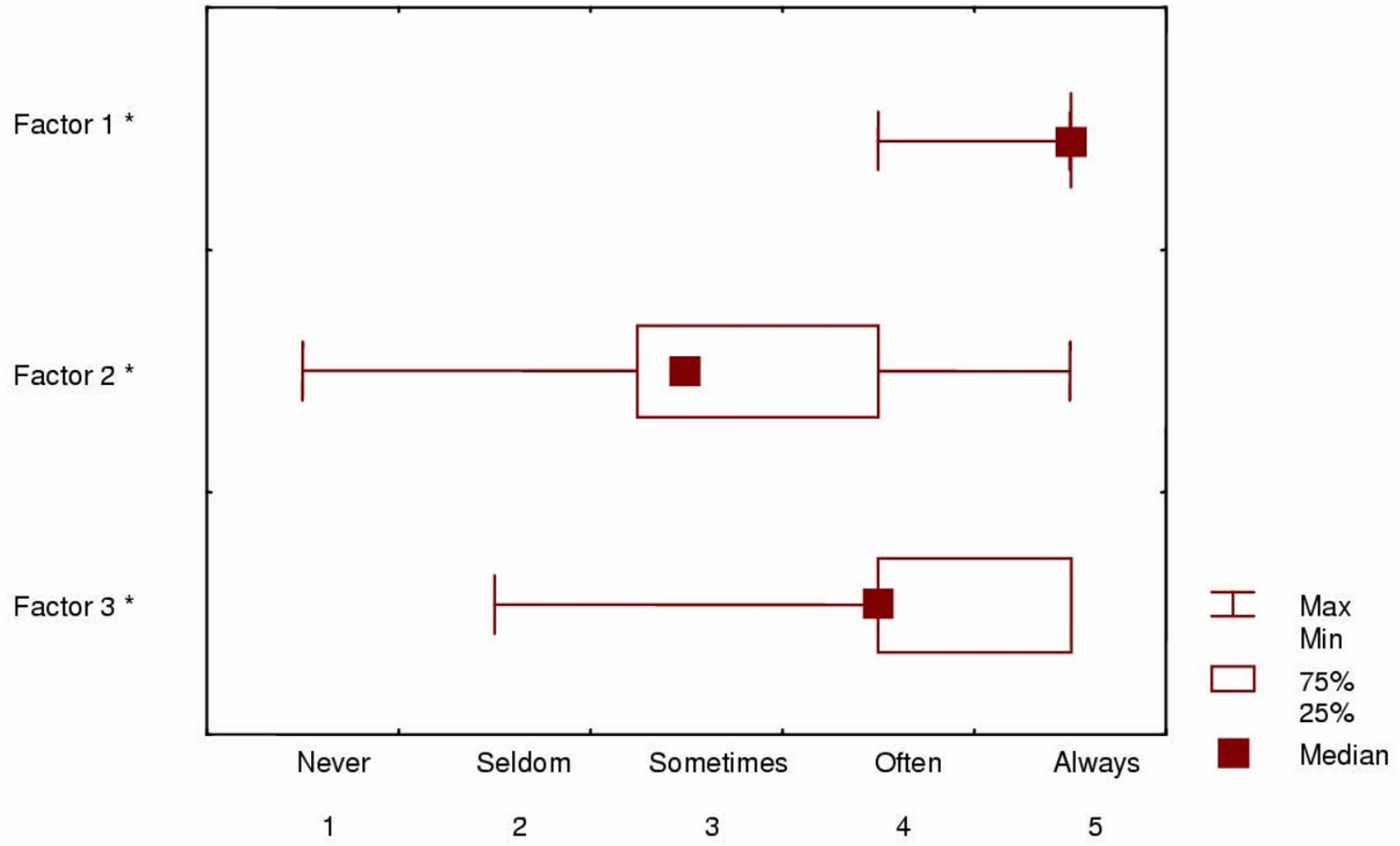

Figure 2

Factors from part I of the questionnaire: comparing scores as defined by factor analysis Part I of the questionnaire $(\mathrm{Q} I)$ is assessing how strong the questionnaire items argue in favour or against antibiotic treatment in case of suspected $\mathrm{RTI}$ in a coughing patient. Factor I includes all the items relating to the lung auscultation. Factor 2 includes only items relating to non-medical reasons, either patient or physician related. Factor 3 includes items determining whether or not there is something unusual happening. Asterisk means that the factor's score significantly differs from the other factors' scores ( $P<0.00 \mathrm{I})$

Items from Q2 not presented in figure 3, and figure 5 respectively (factor loading $\leq .40$ to the yielded three factors) support or counter antibiotic treatment as well. In favour ( median $=1$ ) are crepitations at lung auscultation, medical history of COPD, onset of new complaints in a viral syndrome, consulting for the second time, dyspnoea, tachypnoea, localised thoracic pain, painful teeth or sinuses, coloured sputum, haemoptysis, reduced vesicular breathing, red throat with exudate on the tonsils, the patient being older than 60 years of age and not consulting rapidly. Neutral (median $=0$ ) are smoking, home visit, frequent coughing, no swollen cervical lymph nodes, no localised thoracic pain, medication demand, as well as an RTI epidemic, a dry cough, a red throat without exudate on the tonsils, the patient is known to you, that you make the patient reconsult anyway after 3 to 4 days, that without antibiotic treatment the patient will already reconsult within two days, if not better and bad compliance with antibiotics. Against (median $=-1$ ) are consulting rapidly, influenza-like symptoms, no worsening after two days and not wanting antibiotic treatment. For most items the interquartile range was 1 .

No relation between the response groups characteristics and the scores as defined by factor analyses of Q1 and Q2 was found to be relevant and significant.

\section{Discussion}

This questionnaire study with adequate response[18] enabled us to quantify and condense the focus group determinants and confirmed our focus group finding, that GPs' decisions to prescribe antibiotics are determined by both medical and non-medical reasons.[12]

Neither the internal validity nor the reliability of the questionnaire was formally assessed, but was assumed acceptable: the questionnaire was developed based upon our focus group study and, notwithstanding other factor load- 


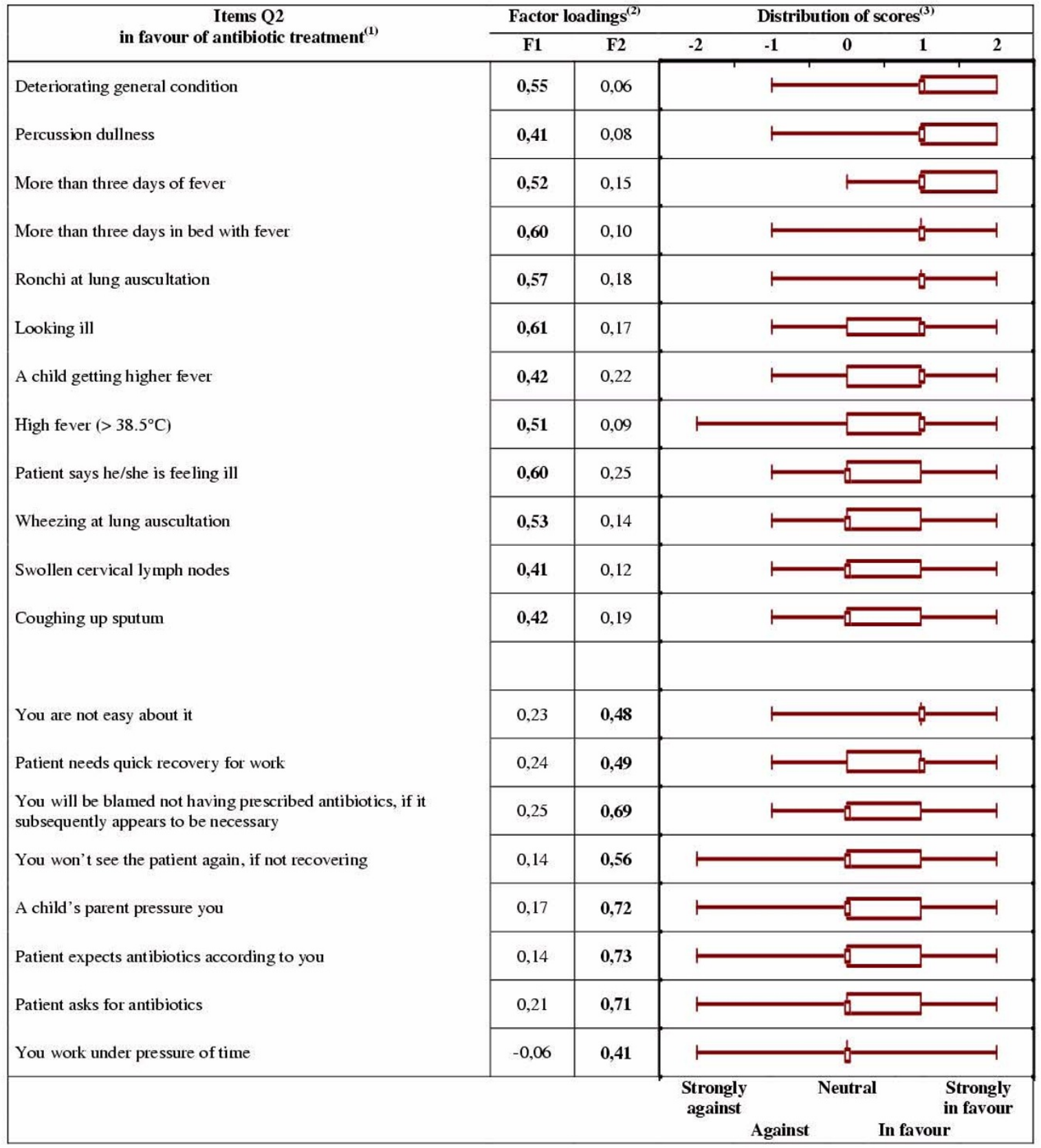

\section{Figure 3}

Items in favour of antibiotic treatment from part 2 of the questionnaire (Q2)( 1 ): distribution of scores and factor loadings per yielded factor (I) Part 2 of the questionnaire (Q2) is assessing how strong the questionnaire items argue in favour or against antibiotic treatment in case of suspected RTI in a coughing patient. Only items that on average argue in favour of antibiotic treatment, with factor loading > .40 to only one of the yielded factors are presented. (2) Factor loadings to the yielded factor are presented. Factor I (FI) only includes items relating to medical reasons, either from the lung auscultation or determining whether or not there is something unusual happening, factor 2 only includes items relating to non-medical reasons, either patient or physician related. (3) The small box represents the median, the larger box the interquartile range, the wiskers the scoring range. 


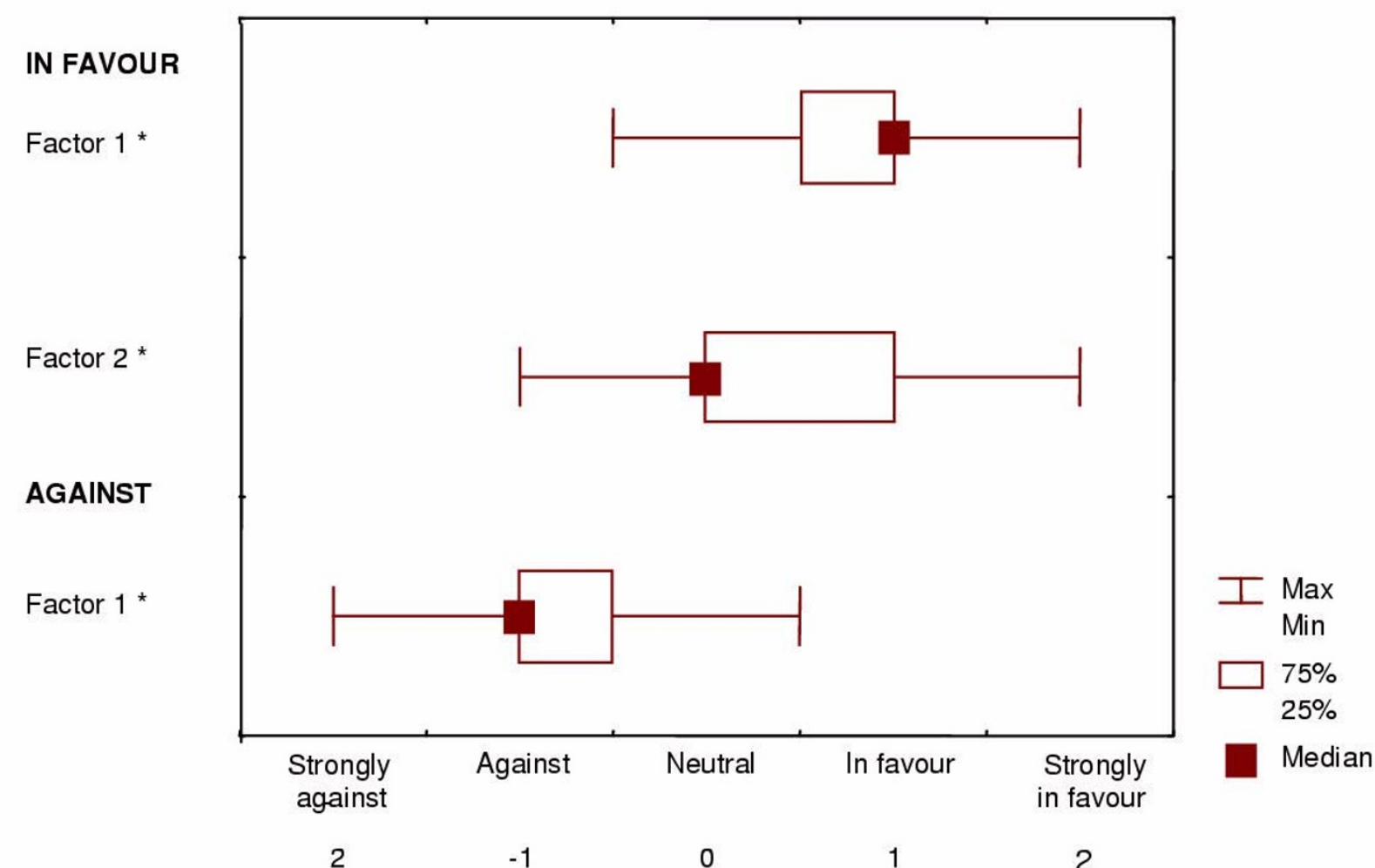

\section{Figure 4}

Factors from part 2 of the questionnaire: comparing scores as defined by factor analysis Part 2 of the questionnaire (Q2) is assessing how strong the questionnaire items argue in favour or against antibiotic treatment in case of suspected $\mathrm{RTI}$ in a coughing patient. Factors 'in favour' include items which according to their mean and sumscore support antibiotic treatment. Factor I only includes items relating to medical reasons, either from the lung auscultation or determining whether or not there is something unusual happening, factor 2 only includes items relating to non-medical reasons, either patient or physician related. Factors 'against' include items which according to their mean and sumscore fail to support antibiotic treatment. Factor I only includes items expressing no need for antibiotic treatment, either medical or non-medical. Asterisk means that the factor's score significantly differs from the other factors' scores $(p<0.00 \mathrm{I})$

ings, the factor analysis of a sample of all GPs in professional training in June 1999 yielded the same results [Coenen, unpublished]. The results of the factor analysis thus seem independent of selection bias. The quantification results though may be biased due to the recruitment and non-response of GPs. The response group characteristics however approximate that of all Flemish GPs, and for a postal survey of general practitioners a response rate of $59.5 \%$ is good according to the literature.[18] Self report also might have limited our data by underestimating the importance of the non-medical reasons. Nevertheless our data show that non-medical reasons determine antibiotic prescribing as well.

In their decision making Flemish GPs seem to consider all the determinants included in the questionnaire. Since the complexity of the prescribing decision, we were not surprised the yielded factors explained only little variance. Nevertheless, the GPs almost always consider the operational factor 'lung auscultation', often 'whether or not there is something unusual happening' - both medical reasons - and to a lesser extent 'non-medical reasons', either patient or physician related. According to the GPs non-medical as well as medical reasons are in favour of antibiotic treatment, the non-medical reasons to a lesser extent.

Yet, for patients with acute (productive) cough the benefit from antibiotics is limited: antibiotics do not influence the duration of productive cough, nor that of limitation in work or activities; out of every 10 patients with acute (productive) cough more than 8 will be clinically improved af- 


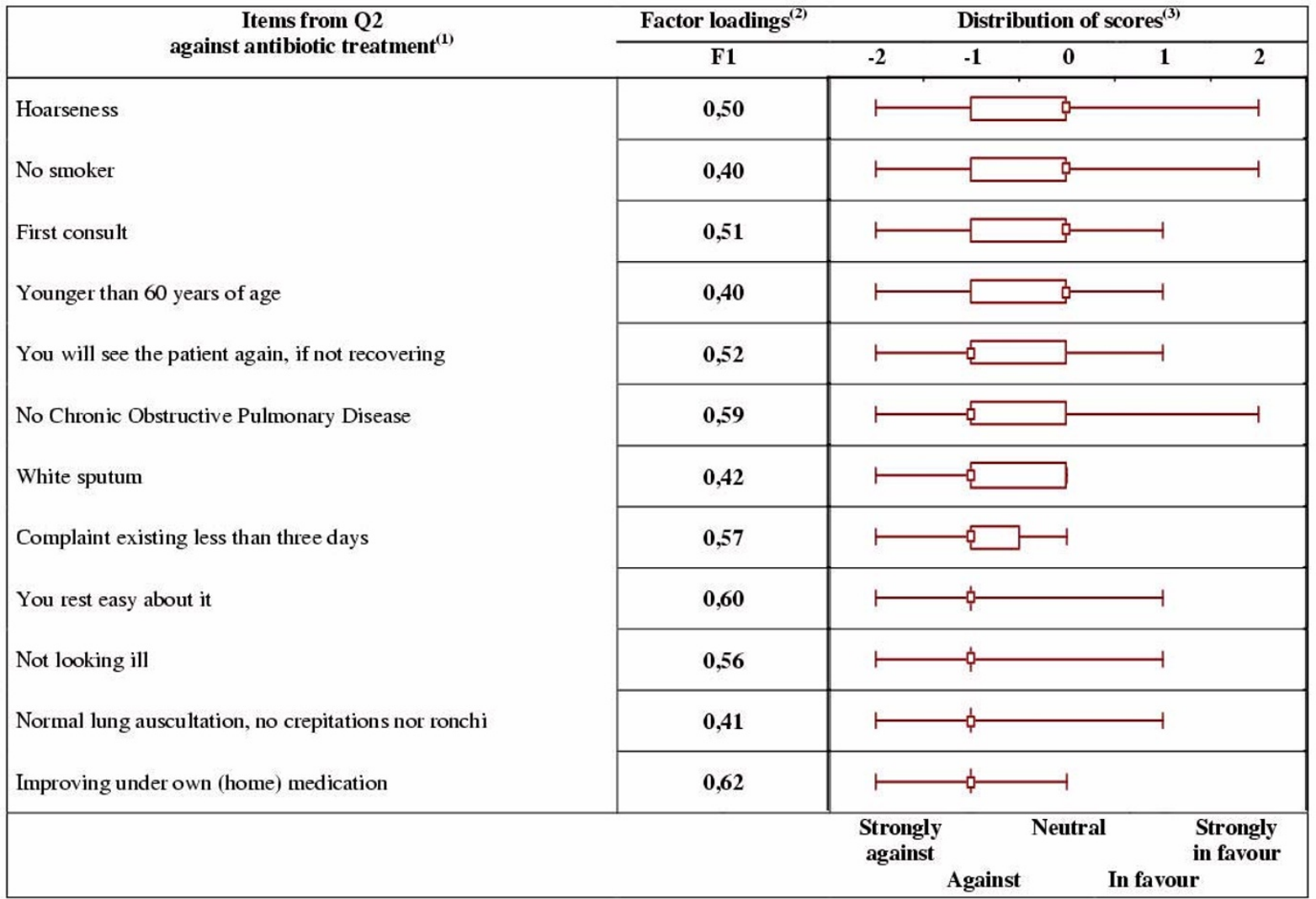

\section{Figure 5}

Items against antibiotic treatment from part 2 of the questionnaire (Q2)(1): distribution of scores and factor loadings per yielded factor (I) Part 2 of the questionnaire is assessing how strong the questionnaire items argue in favour or against antibiotic treatment in case of suspected RTI in a coughing patient. Only items that on average argue against antibiotic treatment, with factor loading $>.40$ to the yielded factor are presented. (2) Factor loadings to the yielded factor are presented. Factor I (FI)only includes items expressing no need for antibiotic treatment, either medical or non-medical. (3) The small box represents the median, the larger box the interquartile range, the wiskers the scoring range.

ter 7-11 days regardless the use of antibiotics; less than one patient extra will be improved due to antibiotics, but as many patients will experience the side effects of treatment. $[9,19]$ And although there is a strong association between focal chest signs and radiographic pneumonia, which suggests presence of focal chest signs may be an important medical reason for antibiotic prescribing, there are no clinical criteria to identify subsets of patients who are most likely to benefit form antibiotic treatment.[20] The presence of focal chest signs however is associated with antibiotic prescribing.[21]

Also non-medical reasons such as patient expectations have been shown to affect prescribing behaviour of GPs for both upper [22] and lower [5] RTIs. And, it has been suggested that GPs' perception of patient expectations may be the strongest determinant for antibiotic prescribing.[23,24] In addition there is little agreement between patient expectations and GPs' perception of these.[24,25] And, for as long as it is difficult in the primary care setting to identify patients for whom antibiotics will be beneficial, these non medical reasons will inevitably keep on playing an important role in the decision to prescribe antibiotics.[26]

Hence good clinical practice guidelines and interventions to optimise antibiotic prescribing for acute cough in Flem- 
ish general practice have to take non-medical reasons into account.

In the Flemish guideline for acute cough, for example, we recommend a clinical and stepwise approach to assess the cause of acute cough. First, possibly life-threatening, treatable conditions such as life-threatening pneumonia should be ruled out. [27-29] Although this first step may automatically and quickly be undertaken, we like to explicitly stress its importance. Next, we would like awareness of other not immediately life threatening conditions. Asthma, postnasal drip or gastro-oesophageal reflux are not as prevalent as an RTI, but require specific treatment.[27] Even though such conditions may not be obvious in a first encounter, it is worthwhile to take them into account. If finally an RTI seems to be the most likely cause, there are no clinical criteria to determine 'which patient will benefit from antibiotics'.[13] Nevertheless, this is the question GPs are confronted with.[12] Because the benefits from antibiotics are outweighed by their harm - side effects, financial cost and bacterial resistance - we promote reassurance, information and treatment without antibiotics in case of suspicion of an RTI. To support this treatment decision, we recommend to involve the patient and to make the non-medical reasons explicit.

In a cluster randomised controlled trial we evaluate whether an educational intervention based on the Flemish guideline for acute cough optimises antibiotic prescribing for acute cough, i.e. achieves the goals of the Belgian public campaign: "Antibiotics, use them less often, but better," ( [http://www.red-antibiotica.org/english/index.html]) without affecting the patients' symptom resolution.[30] We focus on the non-medical reasons for prescribing, more specifically on the GP's perception of patient expectations. Using the baseline data of this controlled before and after study we will also validate the importance of determining whether there is something unusual happening, the lung auscultation, and non-medical reasons in the prescribing decision of Flemish GPs.

\section{Conclusions}

This study assessed the importance for the antibiotic prescribing decision of the determinants of previous focus group research and confirmed it's findings.[12] According to the GPs non-medical as well as medical reasons can argue in favour of antibiotic treatment. Good clinical practice guidelines and interventions to optimise antibiotic prescribing have to take non-medical reasons for antibiotic prescribing into account.

\section{Competing interests}

SC is research assistant of the Fund for Scientific ResearchFlanders. The study was funded by a research grant for small projects of the University of Antwerp. The sponsors of the study had no role in study design, data collection, data analysis, data interpretation, or writing of the report.

\section{Authors' contributions}

SC conceived of the study, participated in its design and carried out the coordination, performed the statistical analysis, and drafted the manuscript. BM participated in the statistical analysis and writing the manuscript. PVR participated in the design of the study, its the coordination and writing the manuscript. JCVDR participated in the designs of the study and the statistical analysis. JD participated in the design of the study and writing the manuscript.

All authors read and approved the final manuscript.

\section{Additional material}

\section{Additional files}

The original questionnaire in Dutch This file contains the original questionnaire used in the current study.

Click here for file

[http://www.biomedcentral.com/content/supplementary/1471-

2296-3-16-s1.doc]

\section{Acknowledgements}

Thanks are due to the University of Antwerp, the Fund for Scientific Research - Flanders and all participating GPs. We also thank the General Practice Respiratory Infections Network (GRIN) members who reviewed and enjoyed earlier drafts of this paper.

\section{References}

I. Cars O, Mölstad S, Melander S: Variation in antibiotic use in the European Union. Lancet 2001, 357:1851-1853

2. Wise $R$, Hart $T$, Cars $O$, Streulens $M$, Helmuth $R$, Huovinen $P$, Sprenger M: Antimicrobial resistance. Is a major threat to public health [editorial]. BMJ 1998, 3 I 7:609-610

3. Howie JG: Clinical judgement and antibiotic use in general practice. $B M J$ 1976, 2:106I-1064

4. Kuyvenhoven M, de Melker R, van der Velden K: Prescription of antibiotics and prescribers' characteristics. A study into prescription of antibiotics in upper respiratory tract infections in general practice. Fam Pract 1993, 10:366-370

5. Macfarlane J, Holmes W, Macfarlane R, Britten N: Influence of patients' expectations on antibiotic management of acute lower respiratory tract illness in general practice: questionnaire study. BMJ 1997, 3 15:121 I-1214

6. Coenen S, Kuyvenhoven M, Butler C, Van Royen P, Verheij T: Variation in European antibiotic use. Lancet 200I, 358:I272

7. De Sutter Al, De Meyere MJ, De Maeseneer JM, Peersman WP: Antibiotic prescribing in acute infections of the nose or sinuses: a matter of personal habit? Fam Pract 200I, I 8:209-2I3

8. Okkes I, Oskam S, Lamberts H: Van klacht naar diagnose [From complaint to diagnosis]. Bussum: Coutinho; 1998

9. Fahey T, Stocks N, Thomas T: Quantitative systematic review of randomised controlled trials comparing antibiotic with placebo for acute cough in adults. $B M$ J 1998, 3 I 6:906-910

10. Butler C, Rollnick S, Kinnersley P, Jones A, Stott N: Reducing antibiotics for respiratory tract symptoms in primary care: consolidating 'why' and considering 'how'. Br J Gen Pract 1998, 48:1865-1870 
1I. Coenen S, van Royen P, Denekens ]: Reducing antibiotics for respiratory tract symptoms in primary care: 'why' only sore throat, 'how' about coughing? [letter]. Br J Gen Pract 1999, 49:400-40।

12. Coenen S, Van Royen P, Vermeire E, Hermann I, Denekens J: Antibiotics for coughing in general practice: a qualitative decision analysis. Fam Pract 2000, 17:380-385

13. Metlay J, Kapoor W, Fine M: Does This Patient Have Community-Acquired Pneumonia? Diagnosing Pneumonia by History and Physical Examination. JAMA 1997, 278: 1440-1445

14. Kassirer J: Our stubborn quest for diagnostic certainty. A cause of excessive testing. NEJM |989, 320:|489-|49|

15. Butler CC, Rollnick S, Pill R, Maggs-Rapport F, Stott N: Understanding the culture of prescribing: qualitative study of general practitioners' and patients' perceptions of antibiotics for sore throats. $B M J$ 1998, 317:637-642

16. Huovinen $\mathrm{P}$, Cars $\mathrm{O}$ : Control of antimicrobial resistance: time for action. The essentials of control are already well known [editorial]. BM] 1998, 317:613-614

17. Michiels B, Avonts D, Van Royen P, Denekens J, Vander Auwera J: A comparison of the upper respiratory tract infection rate between general practitioners and their patients. Eur J Epid 2001

18. McAvoy B, Kaner E: General practice postal surveys: a questionnaire too far? $B M]$ 1996, 31 3:732-733

19. Smucny J, Fahey T, Becker L, Glazier R, Mclsaac W: Antibiotics for acute bronchitis. In: The Cochrane Library Oxford: Update Software 2002

20. Macfarlane J, Holmes W, Gard P, Macfarlane R, Rose D, Weston V, Leinonen M, Saikku P, Myint S: Prospective study of the incidence, aetiology and outcome of adult lower respiratory tract illness in the community. Thorax 2001, 56:109-1I4

21. Macfarlane J, Lewis SA, Macfarlane R, Holmes W: Contemporary use of antibiotics in 1089 adults presenting with acute lower respiratory tract illness in general practice in the U.K.: implications for developing management guidelines. Respir Med 1997, $91: 427-434$

22. Little P, Williamson I, Warner G, Gould C, Gantley M, Kinmonth AL: Open randomised trial of prescribing strategies in managing sore throat. BM] | 997, 3 | 4:722-727

23. Cockburn J, Pit S: Prescribing behaviour in clinical practice: Patients' expectations and doctors' perceptions of patients' expectations - a questionnaire study. BM/ 1997, 3 I 5:520-523

24. Britten $N$, Ukoumunne $O$ : The influence of patients' hopes of receiving a prescription on doctors' perceptions and the decision to prescribe: a questionnaire survey. $B M]$ I997, 3 I 5:15061510

25. Dosh S, Hickner J, Mainous Al, Ebell M: Predictors of antibiotic prescribing for nonspecific upper respiratory tract infections, acute bronchitis, and acute sinusitis. J Fam Pract 2000, 49:407-4I4

26. Fahey $\mathrm{T}$ : Antibiotics for respiratory tract symptoms in general practice. BrJ Gen Pract 1998, 48:1815-1816

27. Managing Cough as a Defense Mechanism and as a Symptom. A Consensus Panel Report of the American College of Chest Physicians. CHEST 1998, II 4: I33S-I8IS

28. Huchon G, Woodhead M: Management of adult community-acquired lower respiratory tract infections. Eur Respir Rev 1998 , 8:39I-426

29. BTS Guidelines for the Management of Community Acquired Pneumonia in Adults. Thorax 200I, 56: I iv-64iv

30. Coenen S, Van Royen P, Michiels B, Denekens J: Promotion of rational antibiotic use in Flemish general practice: implementation of a guideline for acute cough [abstract]. Prim Care Respir J 2002, II :56

\section{Pre-publication history}

The pre-publication history for this paper can be accessed here:

http://www.biomedcentral.com/1471-2296/3/16/prepub
Publish with BioMed Central and every scientist can read your work free of charge

"BioMedcentral will be the most significant development for disseminating the results of biomedical research in our lifetime." Paul Nurse, Director-General, Imperial Cancer Research Fund

Publish with BMC and your research papers will be:

- available free of charge to the entire biomedical community

- peer reviewed and published immediately upon acceptance

- cited in PubMed and archived on PubMed Central

- yours - you keep the copyright

Submit your manuscript here:

http://www.biomedcentral.com/manuscript/
BioMedcentral.com editorial@biomedcentral.com 